\title{
miRNAs being Key Contributors to Chronic Pain Development and Maintenance
}

\author{
Dr. Swaroopa. Maralla ${ }^{* 1}$, D. Bharathi ${ }^{2}$ \\ ${ }^{1}$ Post Doc Fellow in Biomedicine, Division of Zoology, Department of Sericulture, Sri Padmavati Mahila University, Tirupati- \\ 517502, Andhra Pradesh, India \\ ${ }^{2}$ Professor, Department of Sericulture, Sri Padmavati Mahila University, Tirupati-517502, Andhra Pradesh, India
}

\begin{abstract}
RNAs, due to their regulatory role in protein expression have a key role in a number of physiological and pathophysiological processes associated with peripheral and central sensitization mechanisms in the nervous system after nociceptive insults. Maladaptive changes in the levels of these proteins may contribute to abnormal synaptic transmission and neuronal intracellular signalling underlying the onset and development of neuropathic pain. The potential of miRNA profiles to serve as biomarkers of pain is emphasized considering the involvement of inflammatory mediators in inflammation-induced changes in miRNA expression. Such changes are considered to contribute to chronic pain development and maintenance. Therefore, miRNAs may be potential targets for the prevention and/or treatment of chronic inflammatory pain. Understanding of these mechanisms may enable the development of novel therapeutic strategies for preventing and/or treating chronic pain.
\end{abstract}

Keywords: miRNAs, pathophysiology, inflammation, therapeutic strategies.

\section{Introduction}

Chronic pain is a major public health concern worldwide with catastrophic consequences affecting the quality of life (QoL). Chronic pain is usually caused by inflammation and tissue or nerve injury characterized by continuousor intermittent burning sensation, hypersensitivity to noxious stimuli (hyperalgesia), and pain in response to normally innocuous stimuli which is normally not painful (allodynia). Chronic pain can be much prolonged and outlast the period of initial injury or damage (e.g., on inflammation or nerve damage) and aggravate the disease burden, deteriorate quality of life and necessitate medical attention.

Recent investigation point the emergence of ncRNAs as key post-transcriptional regulators to target several mediators in pain pathways simultaneously by manipulating the expression and function of a single ncRNA. One of the most important reasons for their therapeutic aptitude is their ability to modulate the stability of a vast number of gene transcripts and ability to influence the expression of a multitude of mRNA targets and the corresponding proteins. Among ncRNAs, miRNAs are the best studied and are now recognized to be archetypal for the diverse attribute of the nervous system, ranging from development to disease pathology [1-4]. MicroRNAs (miRNAs, miRs) and small interfering RNAs (siRNAs) constitute a class of small noncoding RNAs of 19-25 nucleotides that mediate RNA interference (RNAi) by post-transcriptionally modulating gene expression.

\section{Role of miRNAs in Chronic Pain}

Inflammation and nerve injury induce changes in the expression of receptors, enzymes, ion channels, neurotransmitters, neuromodulators, and structural proteins in primary sensory neurons of the dorsal root ganglion (DRG) at both the transcriptional and translational levels [5-
7]. Such changes are thought to contribute to chronic pain development and maintenance.

An interesting association has been demonstrated very recently between abnormal expression of ncRNAs and the progression of diseases [8,9]. Earlier studies have shown that peripheral inflammation and nerve injury initiate changes in the expression of some miRNAs and Kcna2 AS RNA in DRG [10-13] and these changes might be responsible for inflammation/nerve injury-induced alterations of some painassociated genes, an increase in DRG neuronal excitability, and pain hypersensitivity $[11,13]$. Recent evidence indicates that ncRNAs might be the pivotal player in the mechanisms that underlie the development and maintenance of chronic pain. Though studies on long ncRNAs are still at the early stage, accumulating evidence indicates that they specifically and selectively target their corresponding gene's expression $[13,14]$.

\section{Biogenesis of miRNA}

miRNA biogenesis is a two-step process in humans and includes both nuclear and subsequent cytoplasmic cleavage events performed by two ribonuclease III endonucleases, Drosha and Dicer [15-21]. The miRNA gene is transcribed to produce a primary miRNA (pri-miRNA) that is processed into a precursor miRNA (pre-miRNA) by the endonuclease Drosha and subsequently miRNA duplex (miRNA:miRNA*, passenger strand designated with asterisk) which ultimately releases mature miRNA [22] after transport to the cytoplasm by exportin 5 , a further processing step by Dicer generates mature miRNA.

\section{4. miRNAs in processing of pain}

Altered protein expression is characteristic feature of chronic pain and contributes to the development of longterm hyperexcitability of nociceptive neurons resulting in peripheral and central sensitization[23]. The modifications 


\section{International Journal of Science and Research (IJSR) \\ ISSN (Online): 2319-7064}

Index Copernicus Value (2015): 78.96 | Impact Factor (2015): 6.391

may involve expressional changes of signaling molecules, transmitters, ion channels, or structural proteins. As miRNAs exert anubiquitous influence on gene expression, they may have a key role in the contribution to these changes. Many studies investigating the regulation and function of miRNAs in different models of pathophysiological pain support this hypothesis. For instance, in an inflammatory rat model of CFA-induced muscle pain, the authors suggested that this decrease in miRNAs allows for an upregulation of "pain-related", proteins that facilitated the development of inflammation and allodynia. [10]

\section{5. miRNAs Regulated by Inflammatory Mediators in Chronic Pain}

In peripheral inflammation or nerve injury, an increase in the inflammatory mediators such as interleukin (IL)- $1 \beta$ causes a change in the expression of miRNAs in dorsal root ganglion (DRG) neurons. This change includes up-regulation of some miRNAs (e.g., miR-7a) and down-regulation of other miRNAs (e.g., miR-21), resulting in an alteration in painrelated genes, such as an increase in $\beta$-subunit of voltagegated sodium channels (Nav), in DRG. Such an alteration leads to an increase in DRG neuronal excitability, spinal central sensitization, and pain hypersensitivity (hyperalgesia and allodynia) concluding the part of microRNAs (miRNAs) contribution to chronic inflammatory and neuropathic pain.

\section{6. miRNAs in acute and inflammatory nociception}

Since peripheral inflammation and nerve injury change the expression of many other genes in addition to Kcna2 in painrelated regions [5-7], it is very likely that those genes, like Kcna2, are also regulated by a corresponding long ncRNAs. Significant regulations of long ncRNA transcription in conditions of peripheral inflammation and nerve injury may be a general cellular response which results in the induction and maintenance of chronic pain. As the long ncRNAs have the character of specifically and selectively targeting the corresponding genes, it is probable that the implication of long ncRNAs in chronic pain will become even more apparent in future.

\section{Scope of miRNAs}

miRNAs represent a new class of pain mediators. miRNA can relieve different types of chronic pain conditions. Developing miRNA-based therapies for the treatment of chronic pain may be feasible. However, a major setback in the process is delineating the peripheral and central mechanisms and contributions of the miRNA-mediated changes that lead to chronic pain. This is especially challenging given the wide range of expression of many known miRNAs. The second important challenge is to develop noninvasive means of delivering miRNA modulators, such as AMOs or mimics without significant off-target effects into DRGs, the brain, and/or the SDH. Developing ncRNA-based therapies for pain treatment remains a challenge because the nervous system is still an appalling ground for considerable manipulations.
Nevertheless, several recent studies provide advances and hope.

\section{Conclusion}

miRNAs or the genes they modulate can be direct targets for future therapeutic interventions. Various diseases are associated with exclusive expression of microRNAs (miRNAs), that reveal deep insights into disease pathology. Since aberrant miRNA expression is a typical feature in a variety of human diseases, an understanding of the gene regulation events in chronic pain mediated by miRNAs could provide avenue for the identification of biomarkers and discovery of novel therapeutic targets. A better understanding of the molecular mechanisms resulting in these maladaptive responses can help develop novel therapeutic strategies and biomarkers for neuropathic pain.

\section{Acknowledgement}

The corresponding author thanks the UGC for providing financial assistance towards Post Doctoral Research.

\section{References}

[1] A.A. Aboobaker, "Drosophila microRNAs exhibit diverse spatial expression patterns during embryonic development", Proc. Natl. Acad. Sci. U.S.A.;102:18017-18022, 2005.

[2] U.Hengst, “ Functional and selective RNA interference in developing axons and growth cones," J. Neurosci.;26:5727-5732, 2006.

[3] R.J. Johnston Jr,"MicroRNAs acting in a doublenegative feedback loop to control a neuronal cell fate decision," Proc. Natl. Acad. Sci. U.S.A, ;102:12449 12454, 2005.

[4] G.M. Schratt, "A brain-specific microRNA regulates dendritic spine development," Nature. ;439:283-289, 2006.

[5] J.N.Campbell, and R.A.Meyer,"Mechanisms of neuropathic pain," Neuron.52:77-92, 2006.

[6] A. Latremoliere, and C.J. Woolf,"Central sensitization: a generator of pain hypersensitivity by central neural plasticity,". J.Pain. 10:895-926, 2009.

[7] W.Wang, J.Gu,Y.Q. Li, and Y.X .Tao,"Are voltagegated sodium channels on the dorsal root ganglion involved in the development of neuropathic pain?," Mol.Pain. 7:16, 2011.

[8] M.Fabbri, and G.A.Calin, "Epigenetics and miRNAs in human cancer,"Adv.Genet. 70:87-99, 2010.

[9] T.A.Farazi, J.I.Spitzer,P. Morozov, and T.Tuschl,"miRNAs in human cancer," J.Pathol. 223:102-115, 2011.

[10] G.Bai, R.Ambalavanar, D.Wei, and D.Dessem, "Downregulation of selective microRNAs in trigeminal ganglion neurons following inflammatory muscle pain," Mol.Pain. ;3:15, 2007.

[11] A.Sakai,F. Saitow, N.Miyake, K. Miyake, T. Shimada, and H. Suzuki,"miR-7a alleviates the maintenance of neuropathic pain through regulation of neuronal excitability," Brain. 136:2738-2750, 2013.

\section{Volume 6 Issue 1, January 2017




\section{International Journal of Science and Research (IJSR) \\ ISSN (Online): 2319-7064}

Index Copernicus Value (2015): 78.96 | Impact Factor (2015): 6.391

[12] A.Sakai, andH.Suzuki, "Nerve injury-induced upregulation of miR-21 in the primary sensory neurons contributes to neuropathic pain in rats," Biochem. Biophys. Res. Commun. 435:176-181, 2013.

[13]X.Zhao, Z.Tang, H.Zhang, F.E.Atianjoh,J.Y. Zhao, L.Liang, W.Wang, X.Guan, S.C.Kao, V.Tiwari, Y.J.Gao, P.N.Hoffman,H. Cui, M.Li, X.Dong, and Y.X.Tao,"A long noncoding RNA contributes to neuropathic pain by silencing Kcna2 in primary afferent neurons," Nat. Neurosci. 16:1024-1031, 2013.

[14]D.S.Kim, J.O. Choi, H.D. Rim, andH.J. Cho,"Downregulation of voltage-gated potassium channel alpha gene expression in dorsal root ganglia following chronic constriction injury of the rat sciatic nerve,” Brain Res.Mol.Brain Res. 105:146-152, 2002.

[15] A.M.Denli, B.B.Tops, R.H.Plasterk, R.F. Ketting, and G.J. Hannon, "Processing of primary microRNAs by the Microprocessor complex,"Nature. 432:231-235, 2004.

[16]T.Du, and P.D.Zamore,"microPrimer: the biogenesis and function of microRNA," Development.132:46454652, 2005.

[17] R.I .Gregory,K.P.Yan, G.Amuthan, T.Chendrimada, B.Doratotaj, N.Cooch, and R.Shiekhattar,"The Microprocessor complex mediates the genesis of microRNAs," Nature. 432:235-240, 2004.

[18]J.Han, Y.Lee,K.H. Yeom,Y.K. Kim, H.Jin, andV.N.Kim,"The Drosha-DGCR8 complex in primary microRNA processing," Genes Dev. 18:3016-3027, 2004.

[19] G.Hutvagner, J.McLachlan,A.E. Pasquinelli, E. Balint, T. Tuschl, andP.D. Zamore, "A cellular function for the RNA-interference enzyme Dicer in the maturation of the let-7 small temporal RNA," Science. 293:834-838, 2001.

[20] Y.Lee, C.Ahn, J.Han, H.Choi, J.Kim, J.Yim , J.Lee,P. Provost, O.Radmark,S. Kim, and V.N. Kim, "The nuclear RNase III Drosha initiates microRNA processing. Nature," 425:415-419, 2003.

[21] Y.Lee, K.Jeon, J.T. Lee, S. Kim, and Kim VN,"MicroRNA maturation: stepwise processing and subcellular localization," EMBO J. 21:4663-4670, 2002.

[22] D.P.Bartel, and C.Z.Chen,"Micromanagers of gene expression: the potentially widespread influence of metazoan microRNAs," Nat. Rev. Genet. 5:396-400, 2004".

[23] R.R.Ji, T.Kohno, K.A.Moore, and C.J.Woolf. Central sensitization and LTP: do pain and memory share similar mechanisms? Trends Neurosci; 26:696-705, 2003. 